\title{
Stroke and Bleeding Risk in Atrial Fibrillation
}

\author{
Keitaro Senoo, MD, Deirdre Lane, PhD, and Gregory YH Lip, MD \\ University of Birmingham Centre for Cardiovascular Sciences, City Hospital, Birmingham, United Kingdom
}

Non-valvular atrial fibrillation (AF) is the most common cardiac arrhythmia in the clinical setting. AF increases both the risk and severity of strokes, and is associated with substantial morbidity and mortality. Despite the clear net clinical benefit of oral anticoagulants (OACs) in patients with AF at risk for stroke, major bleeding events, especially intracranial bleeds, may be devastating. In the last decade, four new OACs have been approved for stroke prevention in patients with AF and are at least as effective as warfarin with better bleeding profiles. These new agents have changed and simplified our approach to stroke prevention because the threshold for initiation of OACs is lowered. An important clinical practice shift is the initial identification of "low-risk" patients who do not need antithrombotic therapy, with low-risk comprising $\mathrm{CHA}_{2} \mathrm{DS}_{2}-$ VASc \{Congestive heart failure, Hypertension, Age $\geq 75$ years (double), Diabetes mellitus, previous Stroke/transient ischemic attack/thromboembolism (double), Vascular disease, Age 65-74 years, and female gender (score of 0 for males and 1 for female)\}. Subsequent to this step, effective stroke prevention consisting of OACs can be offered to patients with one or more stroke risk factors. Apart from stroke risk, another consideration is bleeding risk assessment, with a focus on the use of the validated HAS-BLED \{Hypertension, Abnormal renal/liver function, Stroke, Bleeding history, Labile international normalized ratio (INR), Elderly (age >65 years), drugs or alcohol concomitantly\} score. A high HAS-BLED score can flag patients potentially at risk for bleeding, and alert clinicians to the need for careful review and follow up, and the need to consider potentially correctable bleeding risk factors that include uncontrolled hypertension, labile INRs, concomitant aspirin use, and alcohol excess. (Korean Circ J 2014;44(5):281-290)

KEY WORDS: Atrial fibrillation; Stroke; Hemorrhage; Risk assessment.

\section{Introduction}

Decisions regarding the use of antithrombotic therapy for stroke prevention in patients with atrial fibrillation (AF) require consideration of the risk of stroke as well as the risk of bleeding. ${ }^{1}$ However, many risk factors for stroke are also risk factors for bleeding, ${ }_{1}^{2)}$ highlighting the importance of clinical assessment to determine whether the benefit of an oral anticoagulant (OAC) use outweighs the risk of bleeding. Despite the clear net clinical benefit of OACs in stroke prevention, the occurrence of a major bleeding event may be devastating. ${ }^{3)}$ The decision to use OAC should, therefore, be based on a careful assessment of both stroke and bleeding risk.

Correspondence: Gregory YH Lip, MD, University of Birmingham Centre for Cardiovascular Sciences, City Hospital, Birmingham 131870H, United Kingdom

Tel: 44121 50705080, Fax: 441215544083

E-mail: g.y.h.lip@bham.ac.uk

This is an Open Access article distributed under the terms of the Creative Commons Attribution Non-Commercial License (http://creativecommons. org/licenses/by-nc/3.0) which permits unrestricted non-commercial use, distribution, and reproduction in any medium, provided the original work is properly cited.
The purpose of this review is to provide an overview of stroke and bleeding risk assessment in AF patients. For decisions regarding antithrombotic therapy, the emphasis is on the use of risk stratification schemes, with a focus on the older $\mathrm{CHADS}_{2}$ \{ Congestive heart failure, Hypertension, Age $\geq 75$ years, Diabetes mellitus, and previous Stroke/transient ischemic attack (double) $\}$, and more recently, the $\mathrm{CHA}_{2} \mathrm{DS}_{2}-\mathrm{VASc}$ \{Congestive heart failure, Hypertension, Age $\geq 75$ years (double), Diabetes mellitus, previous Stroke/transient ischemic attack/thromboembolism (double), Vascular disease, Age 65-74 years, and female gender\} and HAS-BLED \{Hypertension, Abnormal renal/liver function, Stroke, Bleeding history, Labile international normalized ratio (INR), Elderly (age>65 years), Drugs or alcohol concomitantly\} scores.

\section{Why Do We Anticoagulate?}

Atrial fibrillation is the most common cardiac arrhythmia encountered in clinical practice. The estimated prevalence of AF is $0.4-1 \%$ in the general adult population ${ }^{4) 5}$ occurring in approximately 2.2 million people in the United States. The prevalence of AF increases to approximately $6 \%$ in people $\geq 65$ years of age and in $10 \%$ of people 
$\geq 80$ years of age. ${ }^{6)}$

Although AF is generally not as immediately life-threatening as ventricular arrhythmias, patients with AF have increased risks of stroke and heart failure, and reduced quality of life. ${ }^{7-9)}$ AF leads to a 5 -fold increased risk of stroke, and it is estimated that up to $25 \%$ of all strokes in the elderly are a consequence of AF. ${ }^{6}$ Furthermore, AFrelated strokes are more severe, with patients twice as likely to be bedridden as patients with stroke from other etiologies and more likely to die. ${ }^{10-12)}$

The mean rate of ischemic stroke among patients with nonvalvu$\operatorname{lar} \mathrm{AF}$ is $5 \%$ per year, which is 2-7 times that of the general adult population. ${ }^{9)}$ The risk of stroke increases from $1.5 \%$ in patients with AF aged $50-59$ years to $23 \%$ in those aged $80-89$ years. ${ }^{11)}$ Antithrombotic therapy, particularly with warfarin, decreases the risk of stroke in patients with $\mathrm{AF}^{13{ }^{13144}}$ Warfarin use is associated with a 64\% risk reduction in stroke and a $26 \%$ reduction in all cause mortality, compared with control or placebo, while aspirin is associated with a non-significant $19 \%$ risk reduction, with no impact on mortality. ${ }^{15)}$

Although AF increases the risk of stroke and thromboembolism 5 -fold, this risk is not homogeneous and is altered by the presence of various stroke risk factors. Thus, risk stratification is important to identify patients with a stroke risk that is significant enough to justify the bleeding risk associated with OACs.

\section{$\mathrm{CHADS}_{2}$ Score}

The $\mathrm{CHADS}_{2}$ score $^{16)}$ is the most commonly used risk score for stroke in AF patients. Despite its common use, several concerns have remained. First, recent studies have not confirmed that the $\mathrm{CHADS}_{2}$ score has good predictive value for 'high risk' individuals. ${ }^{17)}$ Second, several known common risk factors for stroke in AF, particularly an age of 65-74 years, female sex, and vascular disease, are not accounted for in the $\mathrm{CHADS}_{2}$ score. ${ }^{1819)}$ Third, aspirin is recommended for a $\mathrm{CHADS}_{2}$ score of $\mathrm{O}_{1}$ and OACs are recommended for a $\mathrm{CHADS}_{2}$ score $\geq 2$, but either aspirin or OAC is considered appropriate for patients with a $\mathrm{CHADS}_{2}$ score of 1 . Several cohorts have shown that $30-50 \%$ of AF patients have a CHADS, score of 1, implying that a large number of AF patients have no clear recommendation for anticoagulation based on these criteria. ${ }^{20211}$ More recent cohorts have shown that even those with a $\mathrm{CHADS}_{2}$ score of 0 can have an annual stroke rate as high as 3.2\% (which is not 'low risk') and those with a score of 1 can have an annual stroke rate of 8\%. ${ }^{22)}$ Furthermore, the $\mathrm{CHADS}_{2}$ score is inadequate to predict the absence of thromboembolism in a cohort of AF patients without any risk factors followed up for 12 years. ${ }^{23)}$

Given the limitations of relying only on the five risk factors in the $\mathrm{CHADS}_{2}$ score, recent guidelines have introduced modifications so as not to rely only on $\mathrm{CHADS}_{2}$. The 2012 American College of Chest Physicians guidelines recommend initially using the $\mathrm{CHADS}_{2}$ score, but, where the $\mathrm{CHADS}_{2}$ score $=0$, additional non-CHADS 2 risk factors such age 65-74 years, vascular disease and female gender should be taken into consideration. ${ }^{24)} \mathrm{A}$ similar approach has been suggested in the Canadian Cardiovascular Society ${ }^{25)}$ and the Japanese Circulation Society guidelines. ${ }^{26)}$

\section{$\mathrm{CHA}_{2} \mathrm{DS}_{2}$-VASc Score}

Real-world cohort data have provided further information to inform stroke risk. Indeed, the independent predictive values of female sex, age 65-74 years, and vascular disease are now evident from numerous cohorts. ${ }^{2728)}$ In addition, a history of congestive heart failure (the $\mathrm{C}$ in $\mathrm{CHADS}_{2}$ ) has not proven to be a consistent stroke risk factor ${ }^{29)}$ whereas moderate-to-severe systolic impairment is clearly an independent stroke risk factor. ${ }^{30)}$ Thus, the European Society of Cardiology (ESC) guidelines now recommend the use of the $\mathrm{CHA}_{2} \mathrm{DS}_{2}$-VASc score for stroke risk stratification (Table 1 and 2). The most recent guidelines of the Asia Pacific Heart Rhythm Society, ${ }_{1}^{31)}$ American Heart Association/American College of Cardiology/Heart Rhythm Society (AHA/ACC/HRS), ${ }_{1}^{32}$ and the United Kingdom National Institute for Health and Care Excellence (NICE) all recommend use of the $\mathrm{CHA}_{2} \mathrm{DS}_{2}$-VASc score for stroke risk stratification.

Table 1. Stroke risk stratification with the $\mathrm{CHADS}_{2}$ and $\mathrm{CHA}_{2} \mathrm{DS}_{2}-\mathrm{VASC}$ scores

Risk score

\begin{tabular}{ll}
\hline CHADS $_{2}$ score & \\
\hline CHF & 1 \\
\hline Hypertension & 1 \\
\hline Age $\geq 75$ & 1 \\
Diabetes & 1 \\
Stroke or TIA & 2 \\
CHA $_{2}$ DS $_{2}$-VASc score & \\
CHF or LVEF $\leq 40 \%$ Hypertension & 1 \\
\hline Age $\geq 75$ & 1 \\
\hline Diabetes & 2 \\
\hline Stroke/TIA/TE & 1 \\
Vascular disease & 2 \\
\hline Age 65-74 & 1 \\
\hline Sex category (female) & 1 \\
\hline
\end{tabular}

Hypertension: systolic blood pressure $>160 \mathrm{~mm} \mathrm{Hg}$. Vascular disease: prior myocardial infarction, peripheral artery disease, and/or aortic plaque. CHF: congestive heart failure, TIA: transient ischemic attack, LVEF: left ventricular ejection fraction, TE: thromboembolism 
Table 2. One year risk of stroke rate with increasing $\mathrm{CHADS}_{2}$ score and $\mathrm{CHA}_{2} \mathrm{DS}_{2}$-VASc score

\begin{tabular}{lcc}
\hline CHADS ${ }_{2}$ score & Patients $(\mathbf{n = 1 7 3 3 )}$ & $\begin{array}{c}\text { Adjusted stroke } \\
\text { rate (\%/year) }\end{array}$ \\
\hline 0 & 120 & 1.9 \\
1 & 463 & 2.8 \\
\hline 2 & 523 & 4.0 \\
\hline 3 & 337 & 5.9 \\
\hline 4 & 220 & 8.5 \\
\hline 5 & 65 & 12.5 \\
\hline 6 & 5 & 18.2 \\
\hline CHA $_{2}$ DS $_{2}$-VASc score & Patients (n=73538) & Adjusted stroke \\
\hline 0 & 6369 & rate (\%/year) \\
\hline 1 & 8203 & 0.7 \\
\hline 2 & 12771 & 1.5 \\
\hline 3 & 17371 & 2.9 \\
\hline 4 & 13887 & 4.3 \\
\hline 5 & 8942 & 6.5 \\
\hline 6 & 4244 & 10.0 \\
\hline 7 & 1420 & 12.5 \\
\hline 8 & 285 & 14.0 \\
\hline 9 & 46 & 14.1 \\
\hline & & 15.9 \\
\hline
\end{tabular}

In its original validation, the $\mathrm{CHA}_{2} \mathrm{DS}_{2}-\mathrm{VASc}$ score was compared with seven other contemporary stroke risk stratification schemas for 1084 patients in the Euro Heart Survey on AF. The survey data demonstrated reasonable predictive ability for high-risk patients and was good at identifying low-risk patients and categorizing few patients into the moderate-risk category. The $\mathrm{CHA}_{2} \mathrm{DS}_{2}$-VASc score has subsequently been validated in numerous AF populations, most commonly compared with $\mathrm{CHADS}_{2 .}{ }^{33)}$ All studies have consistently confirmed the ability of the $\mathrm{CHA}_{2} \mathrm{DS}_{2}$-VASc score to reliably identify truly low-risk patients, who can be managed with no antithrombotic therapy, as well as to predict stroke and thromboembolism in high-risk patients with AF. Indeed, patients with $<65$ years of age (irrespective of gender) have a very low absolute stroke risk, and a $\mathrm{CHA}_{2} \mathrm{DS}_{2}$-VASc score of 0 (in males) and 1 (in females) identifies these low risk patients as the first decision step, ${ }^{23(34) 35)}$ who may reasonably be considered for no antithrombotic treatment. Subsequent to this step, all other AF patients with one or more stroke risk factors (that is $\mathrm{CHA}_{2} \mathrm{DS}_{2}$-VASc score $=1$ in males, or score $\geq 2$ in all), should be considered for OAC (Fig. 1).

Despite the strong evidence in favor of OAC use for stroke prevention, a recent systematic review ${ }^{36)}$ investigating the current treatment practice for stroke prevention in eligible AF patients revealed the underuse of OACs as treatment (defined as $<70 \%$ of eligible patients receiving $\mathrm{OAC}$ ), particularly among those patients at highest risk (i.e., those with a previous stroke/transient ischemic attack). Overestimation of the risk of bleeding by physicians is a key barrier to OAC prescription ${ }^{37)}$ particularly among elderly patients. In the the Birmingham Atrial Fibrillation Treatment of the Aged Study trial, OACs were beneficial in the elderly with a superior reduction in stroke and thromboembolism. Importantly, there was no significant difference in major bleeding between warfarin and aspirin. ${ }^{38)}$

\section{Who Is at Risk for Bleeding?}

Bleeding risk assessment is complex, and many risk factors for bleeding are also risk factors for stroke. Many risk factors for bleeding have been identified. As recently as 2008, only four bleeding risk scores had been applied to AF populations, and only one score, Hepatic or renal disease, Ethanol abuse, Malignancy, Older age, Reduced platelet count or function, Re-bleeding, Hypertension, Anemia, Genetic factors, Excessive fall risk, and Stroke (HEMORR ${ }_{2} \mathrm{HAGES}$ ) had been derived and validated in an AF population. ${ }^{39)}$

In 2010, the HAS-BLED score (Fig. 2) was first proposed, having been derived and validated in the Euro Heart survey population. ${ }^{40)}$ HAS-BLED score is a simple bleeding risk tool representing each of the following common bleeding risk factors and assigning 1 point for the presence of each: hypertension (uncontrolled systolic blood pressure $>160 \mathrm{~mm} \mathrm{Hg}$ ), abnormal renal and/or liver function, previous stroke, bleeding history or predisposition, labile INRs, elderly, and concomitant drugs and/or alcohol excess. The HAS-BLED scores range from $0-9$, with scores $\geq 3$ indicating high risk of bleeding, for which caution and regular review of the patient are recommended. The HAS-BLED score has been validated in multiple independent populations, where it performed as well as (and sometimes better than) the more complex HEMORR ${ }_{2}$ HAGES score. ${ }^{40) 411}$ A close correlation also exists between an increasing HAS-BLED score and major bleeding or intracranial hemorrhage. ${ }^{27)}$ In one analysis of AF patients receiving anticoagulants, the HAS-BLED score was a good predictor of major bleeding and a modest predictor of cardiovascular events and death. ${ }^{42)}$

In 2011, the AnTicoagulation and Risk Factors in Atrial Fibrillation (ATRIA) bleeding risk score in AF patients receiving anticoagulants was proposed. ${ }^{43)}$ Five independent variables were included in the final model: anemia (hemoglobin $<13 \mathrm{~g} / \mathrm{dL}$ in men and $<12 \mathrm{~g} / \mathrm{dL}$ in women; 3 points), severe renal disease (glomerular filtration rate $<30$ $\mathrm{mL} / \mathrm{min}$ or dialysis dependent; 3 points), age $\geq 75$ years (2 points), prior bleeding (1 point), and hypertension ( 1 point). Collapsed into a 3-category risk score, major bleeding rates were $0.8 \%$ for low-risk (0-3 points), 2.6\% for intermediate-risk (4 points) and 5.8\% for high-risk $(\geq 5)$ patients. The high-risk category effectively concentrated bleeding events such that $42 \%$ of events occurred in $10.2 \%$ 


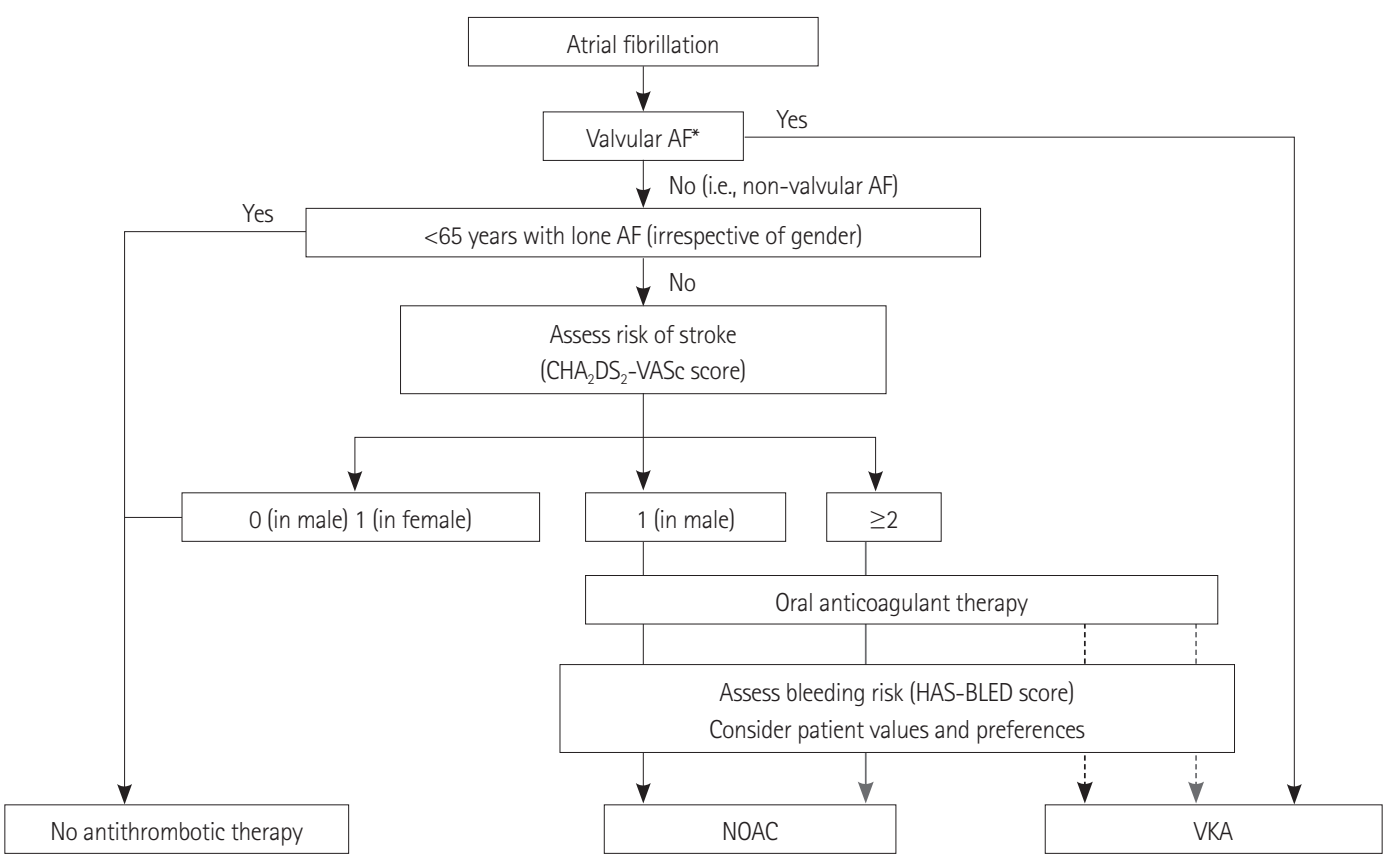

Fig. 1. Flow diagram for stroke prevention based on the 2012 ESC guideline on atrial fibrillation. Antiplatelet therapy with aspirin plus clopidogrel or-, less effectively, aspirin only, should be considered in patients who refuse any OAC, or cannot tolerate anticoagulants for reasons unrelated to bleeding. If there are contraindications to OAC or antiplatelet therapy, left atrial appendage occlusion, closure or excision may be considered. Line: solid: best option, dashed: alternative option. *Includes rheumatic valvular disease and prosthetic valves. AF: atrial fibrillation, $\mathrm{CHA}_{2} \mathrm{DS}_{2}-\mathrm{VASc}$ : congestive heart failure, Hypertension, Age $\geq 75$ years (double), Diabetes mellitus, previous stroke/transient ischemic attack/thromboembolism (double), Vascular disease, age 65-74 years, and female gender, HAS-BLED: hypertension, Abnormal renal/liver function, Stroke, Bleeding history, Labile international normalized ratio (INR), Elderly (age >65 years), drugs or alcohol concomitantly, NOAC: novel oral anticoagulant, VKA: vitamin K antagonist.

\begin{tabular}{lc}
\hline \multicolumn{1}{c}{ Clinical characteristic } & Points \\
\hline Hypertension $(\mathrm{SBP}>160 \mathrm{~mm} \mathrm{Hg})$ & 1 \\
Abnormal renal or liver function & 1 or 2 \\
Stroke & 1 \\
Bleeding & 1 \\
Labile INRs & 1 \\
Elderly (age $>65$ years $)$ & 1 \\
Drugs or alcohol & 1 or 2 \\
Cumulative score & Range $0-9$ \\
\hline
\end{tabular}

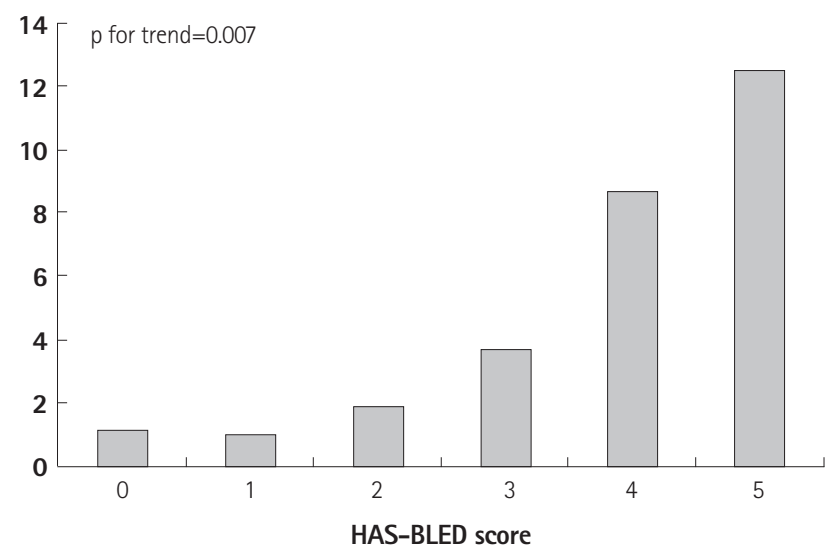

Fig. 2. One year risk of major bleeding with increasing HAS-BLED score. Event rates progressively increased from 1.13\% to $12.5 \%$ in patients with different HAS-BLED scores. Hypertension: uncontrolled systolic blood pressure $>160 \mathrm{~mm} \mathrm{Hg}$, Abnormal renal function: chronic dialysis, renal transplant, or serum creatinine $\geq 200 \mu \mathrm{mol} / \mathrm{L}$, Abnormal liver function: chronic hepatic disease (e.g., cirrhosis) or bilirubin $>2 x$, and serum transaminases $>3 x$, upper limit of normal, Bleeding: previous bleeding requiring hospitalization or causing a decrease in hemoglobin $>2 \mathrm{~g} / \mathrm{L}$ and/or requiring blood transfusion, or predisposition to bleeding such as bleeding diathesis or anemia, Labile INR: time spent within target therapeutic range $<60 \%$, Drugs or alcohol: concomitant use of aspirin, non-steroidal anti-inflammatory drugs or alcohol >20 U/week. INR: international normalized ratio.

of cohort person-years. The low-risk category accounted for 83\% of follow-ups and had an observed bleeding rate $<1 \%$. The c-statistic was 0.74 for the continuous score and 0.69 for the 3-category score. The many limitations of this score have been highlighted and discussed.
HAS-BLED score has been shown to outperform the older HEM$O R R_{2} H A G E S$ and less practical ATRIA scores in predicting clinically relevant bleeding in multiple real-world and trial cohorts. ${ }^{44-46)}$ Thus, the HAS-BLED score is recommended in the ESC guidelines as well as the Canadian Cardiovascular Society guidelines ${ }^{25)}$ and the 2014 
NICE guidelines. The AHA/ACC/HRS guidelines and the JCS guidelines 2014 have also introduced the specific contents of the HASBLED score and implied the importance of the score.

In the ESC guidelines, a HAS-BLED score $\geq 3$ represents sufficient high risk, such that caution and/or regular review of a patient is needed to minimize the risk of complications. It also encourages identification of correctable common bleeding risk factors, such as uncontrolled blood pressure, labile INRs (if on warfarin, to improve time in the therapeutic range), and concomitant aspirin or NSAID use. The determination of such a score allows informed decisions regarding the use of a low-dose or high-dose regime of non-VKA OACs (NOACs, previously referred to as new or novel OACs). ${ }^{47)} \mathrm{Im}$ portantly, a high HAS-BLED should not be a reason to withhold OAC as the net clinical benefit for stroke reduction outweighs the small risk of serious bleeding, but rather to identify those patients in whom caution with such treatment and regular reviews are warranted.

\section{Non-Vitamin K Oral Anticoagulants}

Large scale phase 3 clinical trials have demonstrated efficacy, safety, and convenience of the NOACs as compared to dose-adjusted warfarin (Table 3). ${ }^{48-51)}$ The NOACs occur in two main drug classes: oral direct thrombin inhibitors (e.g., dabigatran) and oral Factor Xa inhibitors (e.g., rivaroxaban, apixaban, edoxaban). All these agents have shown non-inferiority to warfarin therapy, and in some cases, superior efficacy for the primary endpoints of stroke and systemic embolism (dabigatran $150 \mathrm{mg}$ b.i.d. or apixaban) or ischemic stroke (dabigatran $150 \mathrm{mg}$ b.i.d.). Importantly, all the NOACs significantly reduce the risk of hemorrhagic stroke and intracranial hemorrhage compared with warfarin. ${ }^{52)}$ Although all-cause mortality was significantly reduced with apixaban and edoxaban $30 \mathrm{mg}$, a similar trend was also observed in other studies.

Current guidelines recommend that NOACs are preferable to Vitamin $\mathrm{K}$ antagonist (VKA) therapy in the vast majority of patients with nonvalvular AF. ${ }^{125)}$ However, no head-to-head trials have been performed, and indirect comparison analyses do not suggest profound differences in efficacy endpoints among the NOACs. Therefore, it is difficult to provide definitive recommendations regarding which NOACs should be used in which patients. Moreover, high quality anticoagulation control with VKAs is associated with good efficacy and safety with low stroke and bleeding risks. Thus, effective stroke prevention in various guidelines with OACs refers to the use of well-controlled warfarin time in therapeutic range (TRR) $\geq 70 \%$; or NOAC."

While NOACs generally offer many advantages, a clinical dilemma is the prediction of which newly diagnosed non-anticoagulated AF patients would do well on warfarin, with a high TR. This is especially relevant considering the costs of the NOACs and given that the benefits of NOACs over VKAs may be only marginal in those with high TTRs. An ESC position paper ${ }^{53)}$ recommended the use of a simple new SAMe- $\Pi_{2} 2 R_{2}$ \{Sex female, Age $<60$ years, Medical history (more than two comorbidities), Treatment (interacting drugs), Tobacco use (doubled), Race (doubled)\} score incorporating common clinical factors. ${ }^{54-57)}$ This score aids decision-making by identifying those AF patients likely to do well on warfarin (SAMe- $\Pi_{2} R_{2}$ score 0-1) or those more likely to have poor anticoagulation control (SAMe$\Pi_{2} R_{2}$ score $>2$ ). Patients with a SAMe- $\Pi_{2} R_{2}$ score $>2$ would probably be better treated with NOACs as initial therapy or targeted to improve their anticoagulation control if warfarin is used. Indeed, poor anticoagulation control in the initial period following warfarin initiation may lead to an excess of strokes pending stabilization of INR control. ${ }^{58)}$

\section{Balancing Stroke and Bleeding Risk}

On the most simplistic level, the risk of ischemic stroke, which OACs have been attempting to prevent, can be balanced with the risk of the most serious bleeding complication of intracranial hemorrhage. ${ }^{3)} \mathrm{A}$ net clinical benefit analysis ${ }^{59)}$ showed that the only category of AF patients with a negative net clinical benefit after warfarin therapy were those with a $\mathrm{CHA}_{2} \mathrm{DS}_{2}-\mathrm{VASc}$ score of $\mathrm{O}$, reflecting the "truly low-risk" status of such patients. Patients with a high HAS-BLED score $(\geq 3)$ derived an even higher net clinical benefit given that their absolute gain in ischemic stroke reduction far outweighed the small increase in intracranial bleeding. Broadly similar findings were observed by Friberg et al. ${ }^{60)}$ who concluded that warfarin should perhaps be more widely used in AF patients given that the net clinical benefit was in favor of its use in most patients, again with the exception of those with a $\mathrm{CHA}_{2} \mathrm{DS}_{2}$-VASc score of 0 . Siu et al. ${ }^{611}$ found a similarly positive net clinical benefit for warfarin over aspirin, and warfarin over no therapy, in Chinese AF patients with one or more additional stroke risk factors.

Novel oral anticoagulants may provide an even greater net clinical benefit. In a modeling analysis, Banerjee et al. ${ }^{62)}$ showed that in patients with a $\mathrm{CHA}_{2} \mathrm{DS}_{2}$-VASc score of 1 , apixaban and both doses of dabigatran (150 and $110 \mathrm{mg}$ twice daily) had a positive net clinical benefit. All three NOACs (dabigatran, rivaroxaban, and apixaban) appear to offer superior net clinical benefit over warfarin in patients with a $\mathrm{CHA}_{2} \mathrm{DS}_{2}-\mathrm{VASc}$ score $\geq 2$, regardless of bleeding risk. When the risks of both stroke and bleeding are elevated, dabigatran, rivaroxaban, and apixaban appear to have an even greater net clinical benefit than warfarin. 


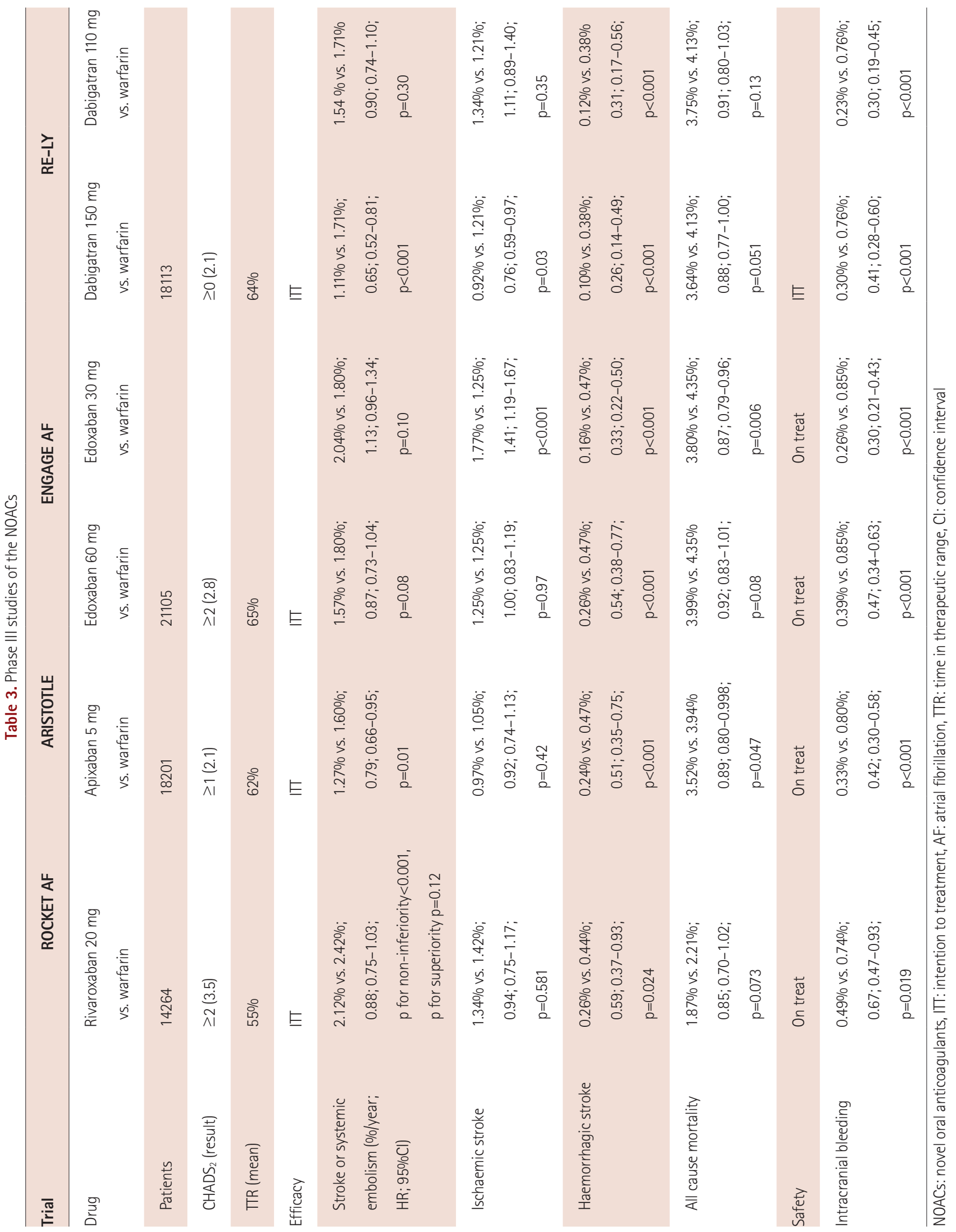




\section{Atrial Fibrillation in the Asian Population}

Both bleeding and thromboembolism rates are generally higher in Asians than non-Asians because of difficulty in proper usage of warfarin in Asians. In a population-based study, Asians had a 2-fold increase in the risk of intracerebral hemorrhage compared to white people. ${ }^{63)}$ Moreover, the risk of intracranial hemorrhage increased to 4-fold compared to white people despite a similar intensity of anticoagulation. ${ }^{64)}$

On the other hand, the risk of stroke and systemic embolism for AF patients taking warfarin appears to be higher in Asians than nonAsians. When Asian and non-Asian patients on warfarin were compared in the RE-LY trial, ${ }_{1}^{5165)}$ Asian patients had a higher stroke rate compared to non-Asians (3.06\% vs. 1.48\%), as well as a poorer average TR (55\%), reflecting poorer quality of anticoagulation control in these patients. In the ROCKET AF trial, ${ }^{48}$ the risk of stroke and systemic embolism was also higher (3.4\% vs. 2.4\%) in East Asians despite the mean $\mathrm{CHADS}_{2}$ score being lower (3.2 vs. 3.47). In the ARISTOTLE trial, ${ }^{4966)}$ the risk of stroke and systemic embolism was also higher in Asians (3.39\% vs. 1.38\%). Overall, Asian physicians tended to keep INR level in a lower range for patients on warfarin due to higher risk of bleeding than non-Asians.

On the basis of these trials, NOACs are preferentially indicated in Asians in terms of both efficacy and safety. Also, some preliminary data suggest that Asian patients with AF might not be the same. ${ }^{67)}$ Thus, future prospective studies are needed for the proper use of NOACs in reference to different ethnic backgrounds.

\section{Non-Pharmacological Approaches}

New devices and systemic therapies have been developed for stroke prevention and are being testing or have been approved for use. In particular, mechanical interventions for stroke prevention have emerged and are being rapidly used. For example, left atrial appendage (LAA) occlusive devices are an alternative treatment strategy for preventing blood clot formation in patients with AF. For patients with AF who are have an ongoing serious bleeding history and/ or are noncompliant (which can be a significant issue for those on warfarin), the possibility of LAA occlusion being an alternative to OACs can be considered.

Several occlusion devices have been developed to exclude the LAA from the systemic circulation, i.e., the Percutaneous Left Atrial Appendage Transcatheter Occlusion (PLAATO; ev3, Plymouth, MN, USA), WATCHMAN (Atritech, Plymouth, MN, USA), and Amplatzer Cardiac Plug devices (AGA Medical, Plymouth, MN, USA). However, the clinical development program for the PLAATO device has been halted, as the device was rather rigid and required up to $50 \%$ oversizing compared with the LAA orifice to achieve a stable position at implantation. ${ }^{68)}$

The WATCHMAN device has been evaluated in a prospective, randomized non-inferiority clinical trial (PROTECT-AF) that compared percutaneous LAA closure and subsequent discontinuation of warfarin with long-term, dose-adjusted warfarin treatment in patients with nonvalvular AF and at least one risk factor for stroke. ${ }^{69)}$ The WATCHMAN device was non-inferior to warfarin with regard to the primary efficacy endpoints-occurrence of ischemic or hemorrhagic stroke, systemic embolism, and cardiovascular death ( $R R=0.71 ; 95 \%$ $\mathrm{Cl}$ 0.44-1.30). However, more primary safety events (excessive major bleeding or procedure-related complications, such as serious pericardial effusion, device embolization, and procedure-related stroke) occurred in the WATCHMAN group ( $\mathrm{RR}=1.53 ; 95 \% \mathrm{Cl} 0.95-2.70){ }^{70)}$ More recently, the ASA Plavix Feasibility Study With WATCHMAN Left Atrial Appendage Closure Technology (ASAP study) reported that LAA closure with the WATCHMAN device can be safely performed without a warfarin transition and is a reasonable alternative in patients at high risk for stroke but with contraindications to systemic OACs. ${ }^{71)}$

Overall, the available data suggest that LAA occlusion reduces the risk of AF-related stroke and might be a promising option, at least for selected patients with AF ineligible for OACs or those who experience severe bleeding complications during treatment with OACs. On the basis of the data available (mostly the PROTECT-AF trial), LAA closure has been given a class Illb recommendation in the 2012 focused update of the ESC AF guidelines.

\section{Quo Vadis?}

Despite the impressive performance of NOACs, some uncertainties exist regarding the new drugs, and more information is needed concerning the long-term anticoagulation with these agents. Also, LAA occlusion reduces the risk of AF-related stroke and might be a promising option, at least for high-risk patients with AF ineligible for OAC or those who experience severe bleeding complications during treatment with OACs. However, LAA occlusion needs to be performed with care by experienced operators because periprocedural complications, such as pericardial effusion or stroke, have been documented. With increased operator experience and technical improvements in LAA occlusion devices, complications can be minimized.

\section{Conclusion}

Decisions regarding appropriate stroke prevention require individual assessment of stroke and bleeding risk on anticoagulation 
with VKA therapy and NOACs. Use of risk scores such as $\mathrm{CHA}_{2} \mathrm{DS}_{2}-$ VASc and HAS-BLED can help in the selection of appropriate antithrombotic agents and management strategies. Also, availability of NOACs offers new possibilities, and these drugs have changed the landscape for stroke prevention in AF.

\section{Acknowledgments}

Keitaro Senoo: Nothing to disclose.

Gregory YH Lip: Consultant for Bayer, Astellas, Merck, AstraZeneca, Sanofi Aventis, Biotronik, BMS/Pfizer, Daiichi-Sankyo, Medtronic and Boehringher Ingelheim. Speakers bureau for Bayer, BMS/Pfizer, Boehringher Ingelheim, Daiichi-Sankyo, Medtronic, and Sanofi Aventis.

Deirdre Lane: Dr. Lane reports investigator-initated grants from Boehringer Ingelheim and Bayer Healthcare, personal fees from BMS, Pfizer, Boehringer Ingelheim and Bayer for educational lectures, non-financial support from Boehringer Ingelheim for conference travel, and royalties from BMJ Clinical Evidence.

\section{References}

1. Camm AJ, Lip GY, De Caterina R, et al. 2012 focused update of the ESC Guidelines for the management of atrial fibrillation: an update of the 2010 ESC Guidelines for the management of atrial fibrillation--developed with the special contribution of the European Heart Rhythm Association. Europace 2012;14:1385-413.

2. Lip GY, Andreotti $F$, Fauchier $L$, et al. Bleeding risk assessment and management in atrial fibrillation patients. Executive Summary of a Position Document from the European Heart Rhythm Association [EHRA], endorsed by the European Society of Cardiology [ESC] Working Group on Thrombosis. Thromb Haemost 2011;106:997-1011.

3. Singer $D E$, Chang $Y$, Fang $M C$, et al. The net clinical benefit of warfarin anticoagulation in atrial fibrillation. Ann Intern Med 2009;151:297-305.

4. Go AS, Hylek EM, Phillips KA, et al. Prevalence of diagnosed atrial fibrillation in adults: national implications for rhythm management and stroke prevention: the AnTicoagulation and Risk Factors in Atrial Fibrillation (ATRIA) Study. JAMA 2001;285:2370-5.

5. Furberg CD, Psaty BM, Manolio TA, Gardin JM, Smith VE, Rautaharju PM. Prevalence of atrial fibrillation in elderly subjects (the Cardiovascular Health Study). Am J Cardiol 1994;74:236-41.

6. Lloyd-Jones D, Adams RJ, Brown TM, et al. Executive summary: heart disease and stroke statistics--2010 update: a report from the American Heart Association. Circulation 2010;121:948-54.

7. Lee WC, Lamas GA, Balu S, Spalding J, Wang Q, Pashos CL. Direct treatment cost of atrial fibrillation in the elderly American population: a medicare perspective. J Med Econ 2008;11:281-98.

8. Thrall G, Lane D, Carroll D, Lip GY. Quality of life in patients with atrial fibrillation: a systematic review. Am J Med 2006;119:448.e1-19.

9. Stewart S, Hart CL, Hole DJ, McMurray JJ. A population-based study of the long-term risks associated with atrial fibrillation: 20-year followup of the Renfrew/Paisley study. Am J Med 2002;113:359-64.
10. Dulli DA, Stanko H, Levine RL. Atrial fibrillation is associated with severe acute ischemic stroke. Neuroepidemiology 2003;22:118-23.

11. Lin HJ, Wolf PA, Kelly-Hayes $M_{1}$ et al. Stroke severity in atrial fibrillation. The Framingham Study. Stroke 1996;27:1760-4.

12. Paciaroni $M$, Agnelli $G$, Caso V, et al. Atrial fibrillation in patients with first-ever stroke: frequency, antithrombotic treatment before the event and effect on clinical outcome. J Thromb Haemost 2005;3:1218-23.

13. Stroke Prevention in Atrial Fibrillation Study. Final results. Circulation 1991;84:527-39.

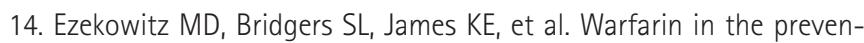
tion of stroke associated with nonrheumatic atrial fibrillation. Veterans Affairs Stroke Prevention in Nonrheumatic Atrial Fibrillation Investigators. N Engl J Med 1992;327:1406-12.

15. Hart RG, Pearce LA, Aguilar MI. Adjusted-dose warfarin versus aspirin for preventing stroke in patients with atrial fibrillation. Ann Intern Med 2007;147:590-2.

16. Hughes M, Lip GY; Guideline Development Group, et al. Stroke and thromboembolism in atrial fibrillation: a systematic review of stroke risk factors, risk stratification schema and cost effectiveness data. Thromb Haemost 2008;99:295-304.

17. Fang $M C$, Go AS, Chang $Y$, et al. Comparison of risk stratification schemes to predict thromboembolism in people with nonvalvular atrial fibrillation. J Am Coll Cardio/ 2008;51:810-5.

18. Benjamin EJ, Levy D, Vaziri SM, D'Agostino RB, Belanger AJ, Wolf PA. Independent risk factors for atrial fibrillation in a population-based cohort. The Framingham Heart Study. JAMA 1994;271:840-4.

19. Kerr $C R$, Humphries K. Gender-related differences in atrial fibrillation. J Am Coll Cardiol 2005;46:1307-8.

20. Gage BF, Waterman AD, Shannon W, Boechler M, Rich MW, Radford MJ. Validation of clinical classification schemes for predicting stroke: results from the National Registry of Atrial Fibrillation. JAMA 2001; 285:2864-70.

21. Nieuwlaat R, Capucci $A$, Lip GY, et al. Antithrombotic treatment in reallife atrial fibrillation patients: a report from the Euro Heart Survey on Atrial Fibrillation. Eur Heart J 2006;27:3018-26.

22. Olesen JB, Torp-Pedersen $C$, Hansen ML, Lip GY. The value of the CHA2DS2-VASC score for refining stroke risk stratification in patients with atrial fibrillation with a CHADS2 score 0-1: a nationwide cohort study. Thromb Haemost 2012;107:1172-9.

23. Potpara TS, Polovina MM, Licina MM, Marinkovic JM, Prostran MS, Lip GY. Reliable identification of "truly low" thromboembolic risk in patients initially diagnosed with "lone" atrial fibrillation: the Belgrade atrial fibrillation study. Circ Arrhythm Electrophysio/ 2012;5:319-26.

24. You JJ, Singer DE, Howard PA, et al. Antithrombotic therapy for atrial fibrillation: Antithrombotic Therapy and Prevention of Thrombosis, 9th ed: American College of Chest Physicians Evidence-Based Clinical Practice Guidelines. Chest 2012;141(2 Suppl):e531S-75S.

25. Skanes AC, Healey JS, Cairns JA, et al. Focused 2012 update of the Canadian Cardiovascular Society atrial fibrillation guidelines: recommendations for stroke prevention and rate/rhythm control. Can J Cardiol 2012;28:125-36.

26. Japanese Circulation Society. Guidelines for Pharmacotherapy of Atrial Fibrillation (JCS 2013). Japan: Japanese Circulation Society;2013. 
27. Friberg L, Rosenqvist M, Lip GY. Evaluation of risk stratification schemes for ischaemic stroke and bleeding in 182678 patients with atrial fibrillation: the Swedish Atrial Fibrillation cohort study. Eur Heart J 2012;33:1500-10.

28. Olesen JB, Lip GY, Lane DA, et al. Vascular disease and stroke risk in atrial fibrillation: a nationwide cohort study. Am J Med 2012;125:826. e13-23.

29. Stroke Risk in Atrial Fibrillation Working Group. Independent predictors of stroke in patients with atrial fibrillation: a systematic review. Neurology 2007;69:546-54.

30. Echocardiographic predictors of stroke in patients with atrial fibrillation: a prospective study of 1066 patients from 3 clinical trials. Arch Intern Med 1998;158:1316-20.

31. Ogawa S, Aonuma K, Tse HF, et al. The APHRS's 2013 statement on antithrombotic therapy of patients with nonvalvular atrial fibrillation. J Arrhythm 2013;29,:90-200.

32. January CT, Wann LS, Alpert JS, et al. 2014 AHA/ACC/HRS Guideline for the Management of Patients With Atrial Fibrillation: A Report of the American College of Cardiology/American Heart Association Task Force on Practice Guidelines and the Heart Rhythm Society. J Am Coll Cardio/ 2014. doi: 10.1016/j.jacc.2014.03.022. [Epub ahead of print]

33. Olesen JB, Lip GY, Hansen ML, et al. Validation of risk stratification schemes for predicting stroke and thromboembolism in patients with atrial fibrillation: nationwide cohort study. BMJ 2011;342:d124.

34. Taillandier $S_{1}$ Olesen JB, Clémenty $N_{1}$ et al. Prognosis in patients with atrial fibrillation and CHA2DS2-VASc Score $=0$ in a community-based cohort study. J Cardiovasc Electrophysiol 2012;23:708-13.

35. Van Staa TP, Setakis E, Di Tanna GL, Lane DA, Lip GY. A comparison of risk stratification schemes for stroke in 79,884 atrial fibrillation patients in general practice. J Thromb Haemost 2011;9:39-48.

36. Ogilvie IM, Newton N, Welner SA, Cowell W, Lip GY. Underuse of oral anticoagulants in atrial fibrillation: a systematic review. Am J Med 2010;123:638-45.

37. Pugh D, Pugh J, Mead GE. Attitudes of physicians regarding anticoagulation for atrial fibrillation: a systematic review. Age Ageing 2011; 40:675-83.

38. Mant J, Hobbs FD, Fletcher $K_{1}$ et al. Warfarin versus aspirin for stroke prevention in an elderly community population with atrial fibrillation (the Birmingham Atrial Fibrillation Treatment of the Aged Study, BAFTA): a randomised controlled trial. Lancet 2007;370:493-503.

39. Lane DA, Lip GY. Use of the CHA(2)DS(2)-VASc and HAS-BLED scores to aid decision making for thromboprophylaxis in nonvalvular atrial fibrillation. Circulation 2012;126:860-5.

40. Pisters R, Lane DA, Nieuwlaat R, de Vos CB, Crijns HJ, Lip GY. A novel user-friendly score (HAS-BLED) to assess 1-year risk of major bleeding in patients with atrial fibrillation: the Euro Heart Survey. Chest 2010; 138:1093-100.

41. Olesen JB, Lip GY, Hansen PR, et al. Bleeding risk in 'real world' patients with atrial fibrillation: comparison of two established bleeding prediction schemes in a nationwide cohort. J Thromb Haemost 2011;9:1460-7.

42. Gallego $P$, Roldán $V$, Torregrosa JM, et al. Relation of the HAS-BLED bleeding risk score to major bleeding, cardiovascular events, and mortality in anticoagulated patients with atrial fibrillation. Circ Arrhythm
Electrophysiol 2012;5:312-8.

43. Fang $M C$, Go AS, Chang $Y$, et al. A new risk scheme to predict warfarinassociated hemorrhage: The ATRIA (Anticoagulation and Risk Factors in Atrial Fibrillation) Study. J Am Coll Cardio/ 2011;58:395-401.

44. Apostolakis S, Lane DA, Guo Y, Buller H, Lip GY. Performance of the HEMORR(2)HAGES, ATRIA, and HAS-BLED bleeding risk-prediction scores in patients with atrial fibrillation undergoing anticoagulation: the AMADEUS (evaluating the use of SR34006 compared to warfarin or acenocoumarol in patients with atrial fibrillation) study. J Am Coll Cardiol 2012;60:861-7.

45. Roldán $V$, Marín $F$, Fernández $H$, et al. Predictive value of the HASBLED and ATRIA bleeding scores for the risk of serious bleeding in a "real-world" population with atrial fibrillation receiving anticoagulant therapy. Chest 2013;143:179-84.

46. Lip GY, Banerjee A, Lagrenade I, Lane DA, Taillandier S, Fauchier L. Assessing the risk of bleeding in patients with atrial fibrillation: the Loire Valley Atrial Fibrillation project. Circ Arrhythm Electrophysiol 2012;5:941-8.

47. Husted $S$, de Caterina $R$, Andreotti $F$, et al. Non-vitamin $K$ antagonist oral anticoagulants (NOACs): No longer new or novel. Thromb Haemost 2014;111:781-2.

48. Patel MR, Mahaffey KW, Garg J, et al. Rivaroxaban versus warfarin in nonvalvular atrial fibrillation. N Eng/ J Med 2011;365:883-91.

49. Granger CB, Alexander JH, McMurray JJ, et al. Apixaban versus warfarin in patients with atrial fibrillation. N Eng/ J Med 2011;365:981-92.

50. Giugliano RP, Ruff CT, Braunwald E, et al. Edoxaban versus warfarin in patients with atrial fibrillation. N Eng/ J Med 2013;369:2093-104.

51. Connolly SJ, Ezekowitz MD, Yusuf S, et al. Dabigatran versus warfarin in patients with atrial fibrillation. N Eng/ J Med 2009;361:1139-51.

52. Potpara TS, Lip GY, Apostolakis S. New anticoagulant treatments to protect against stroke in atrial fibrillation. Heart 2012;98:1341-7.

53. De Caterina $R$, Husted $S$, Wallentin $L$, et al. Vitamin $K$ antagonists in heart disease: current status and perspectives (Section III). Position paper of the ESC Working Group on Thrombosis--Task Force on Anticoagulants in Heart Disease. Thromb Haemost 2013;110:1087-107.

54. Apostolakis S, Sullivan RM, Olshansky B, Lip GY. Factors affecting quality of anticoagulation control among patients with atrial fibrillation on warfarin: the SAMe- $\Pi_{2} R_{2}$ score. Chest 2013;144:1555-63.

55. Poli $D$, Antonucci $E$, Testa $S$, Lip GY. A prospective validation of the SAME-TT2R 2 score: how to identify atrial fibrillation patients who will have good anticoagulation control on warfarin. Intern Emerg Med 2014;9:443-7.

56. Lip GY, Haguenoer K, Saint-Etienne C, Fauchier L. Relationship of the SAME-TT2R2score to poor quality anticoagulation, stroke, clinically relevant bleeding and mortality in patients with atrial fibrillation. Chest 2014. doi: 10.1378/chest.13-2976. [Epub ahead of print]

57. Gallego P, Roldán $V$, Marin F, et al. SAME-T2R2 score, time in therapeutic range and outcomes in anticoagulated patients with atrial fibrillation. Am J Med 2014. doi: 10.1016/j.amjmed.2014.05.023. [Epub ahead of print]

58. Azoulay L, Dell'aniello S, Simon TA, Renoux C, Suissa S. Initiation of warfarin in patients with atrial fibrillation: early effects on ischaemic strokes. Eur Heart J 2013. [Epub ahead of print] 
59. Olesen JB, Lip GY, Lindhardsen J, et al. Risks of thromboembolism and bleeding with thromboprophylaxis in patients with atrial fibrillation: A net clinical benefit analysis using a 'real world' nationwide cohort study. Thromb Haemost 2011;106:739-49.

60. Friberg L, Rosenqvist M, Lip GY. Net clinical benefit of warfarin in patients with atrial fibrillation: a report from the Swedish atrial fibrillation cohort study. Circulation 2012;125:2298-307.

61. Siu CW, Lip GY, Lam KF, Tse HF. Risk of stroke and intracranial hemorrhage in 9727 Chinese with atrial fibrillation in Hong Kong. Heart Rhythm 2014. doi: 10.1016/j.hrthm.2014.04.021. [Epub ahead of print]

62. Banerjee A, Lane DA, Torp-Pedersen C, Lip GY. Net clinical benefit of new oral anticoagulants (dabigatran, rivaroxaban, apixaban) versus no treatment in a 'real world' atrial fibrillation population: a modelling analysis based on a nationwide cohort study. Thromb Haemost 2012; 107:584-9.

63. van Asch CJ, Luitse MJ, Rinkel GJ, van der Tweel I, Algra A, Klijn CJ. Incidence, case fatality, and functional outcome of intracerebral haemorrhage over time, according to age, sex, and ethnic origin: a systematic review and meta-analysis. Lancet Neuro/ 2010;9:167-76.

64. Shen AY, Yao JF, Brar SS, Jorgensen MB, Chen W. Racial/ethnic differences in the risk of intracranial hemorrhage among patients with atrial fibrillation. J Am Coll Cardiol 2007;50:309-15.

65. Hori M, Connolly SJ, Zhu J, et al. Dabigatran versus warfarin: effects on ischemic and hemorrhagic strokes and bleeding in Asians and non-
Asians with atrial fibrillation. Stroke 2013:44:1891-6.

66. Goto S, Zhu J, Lisheng L, et al. Efficacy and safety of apixaban compared with warfarin for stroke prevention in atrial fibrillation in East Asia. Eur Heart J 2013;34(Abstract Supplement):1039.

67. Chiang CE, Wang KL, Lip GY. Stroke prevention in atrial fibrillation: an Asian perspective. Thromb Haemost 2014;111:789-97.

68. Landmesser U, Holmes DR Jr. Left atrial appendage closure: a percutaneous transcatheter approach for stroke prevention in atrial fibrillation. Eur Heart J 2012;33:698-704.

69. Holmes DR, Reddy VY, Turi ZG, Percutaneous closure of the left atrial appendage versus warfarin therapy for prevention of stroke in patients with atrial fibrillation: a randomised non-inferiority trial. Lancet 2009;374:534-42.

70. Reddy VY, Doshi SK, Sievert H, et al. Percutaneous left atrial appendage closure for stroke prophylaxis in patients with atrial fibrillation: 2.3-Year Follow-up of the PROTECT AF (Watchman Left Atrial Appendage System for Embolic Protection in Patients with Atrial Fibrillation) Trial. Circulation 2013;127:720-9.

71. Reddy VY, Möbius-Winkler S, Miller MA, et al. Left atrial appendage closure with the Watchman device in patients with a contraindication for oral anticoagulation: the ASAP study (ASA Plavix Feasibility Study With Watchman Left Atrial Appendage Closure Technology). J Am Coll Cardiol 2013;61:2551-6. 